\title{
GENETIC ANALYSIS SYSTEMS: IMPROVEMENTS AND METHODS
}

\author{
Rolfe C. Anderson, Gregory J. Bogdan, Alex Puski, Xing Su \\ Affymetrix, Inc., 3380 Central Expressway, Santa Clara, CA 95051
}

\begin{abstract}
We have developed integrated genetic analysis systems employing improved structures and methods for manipulating fluids and temperatures, new enzymatic reactions, and increased process density. Enhanced mixing, rapid thermal cycling, and fluidic reliability have been demonstrated. Experiments have revealed a new meniscus-driven mixing phenomenon generally characteristic to liquid-plug flow systems. The integrated fluid and thermal control methods developed in this work can be generally applied to other systems for chemical and biochemical analysis and may be amenable to further miniaturization.
\end{abstract}

\section{INTRODUCTION}

Analytical processes benefit from improved reproducibility and reduced operator intervention as entire sample-preparation processes and analytical steps are combined on a single device. Miniaturization and integration can provide device portability, multiplicity, and potentially low cost. Integration of genetic analysis are particularly valuable because complex multistep assays on multiple samples are often desired.

Reports on the development of miniaturized analysis systems have typically been limited to single reactions followed by analysis $[1,2]$, or have described single operations such as cell lysis $[3,4]$. We previously reported on a device that performs a multistep virus mutation assay, first extracting genetic material from a blood sample, then performing a series of enzymatic reactions, and finally carrying out a hybridization reaction with a polynucleotide array [5].

Polynucleotide GeneChip ${ }^{\circledR}$ arrays enable the rapid parallel monitoring of genetic information through the selective hybridization of nucleic acids. After a prepared sample reacts with the array, regions of binding are detected using fluorescence imaging [6]. GeneChip arrays are fabricated using a photo-directed synthesis technique, where tens of thousands of regions with different polynucleotide probes are created on a glass surface. GeneChip applications include HIV virus polymorphisms [7], gene expression [8], cystic fibrosis [9], cancer-related mutations [10], and other sequence analysis assays. These assays generally include the preparation of a labeled and fragmented polynucleic acids at an

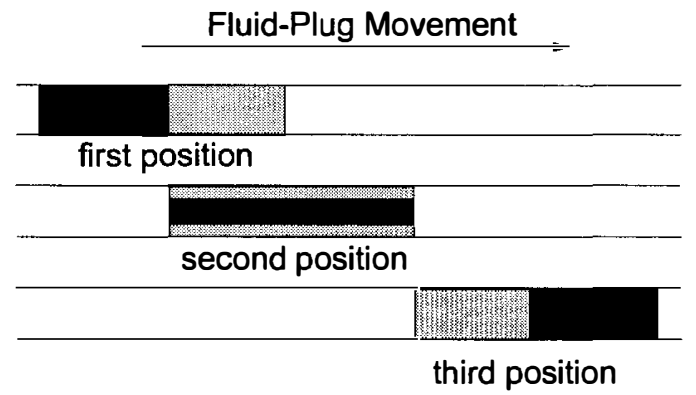

Figure 1. Observed meniscus recirculation mixing. Idealized sketch of dye positions as liquid plug is moved through a channel. appropriate concentration. The required series of bench-top processes include extraction and purification of nucleic acid from a sample, enzymatic amplification of the region of interest (e.g. PCR), followed by fragmentation, and labeling reactions. An automated system incorporating these processes offers the advantages of reduced sample volumes, contamination and operator handling, and better reproducibility.

We previously reported on an entire HIV assay from nucleic acid extraction through hybridization using new methods for positioning, metering, and debubbling microliter-volume fluids without the use of sensors. In this work: a) the ability to manipulate fluids has been greatly enhanced by the development of structures and methods for reliable fluid linking, positioning, and mixing, b) an improved thermal control scheme has been demonstrated with faster cycling and scaleability, c) new enzymatic reactions with applications to other genetic assays such as a cytochrome P450 mutation assay have been demonstrated, and d) cartridges for this assay with multiple sample handling for higher throughput have been designed, fabricated and tested.

\section{MENISCUS RECIRCULATION MIXING}

Homogeneous mixing in reaction systems is generally desirable and has been widely discussed for microfluidics [11] but the hydrodynamics of fluid plugs separated by air has not been represented. In these systems fluid movement at the meniscus can dominate mixing. Flow visualization experiments have revealed a circulation phenomenon that is specific to systems with a moving meniscus. In the experiment, a liquid plug dyed with two different colors is introduced into the channel. As illustrated in Figure 1, moving the fluid plug through a channel causes the dye at the trailing end of a fluid to first penetrate the center of the channel and then encompass the leading end of the fluid plug [12]. As a

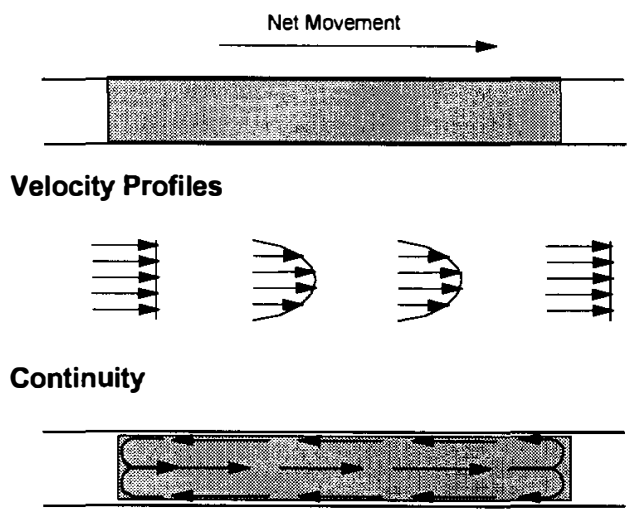

Figure 2. Circulation flow induced by moving liquid plug through a channel. A uniform (plug flow) velocity profile is imposed by the movement of the leading and trailing menisci. Towards the midpoint of the plug, parabolic flow is approached. The requirement of fluid continuity causes the radial inward and outward velocities drawn at the trailing and leading edges, respectively. (Note: last sketch is drawn in Eulerian reference frame). 


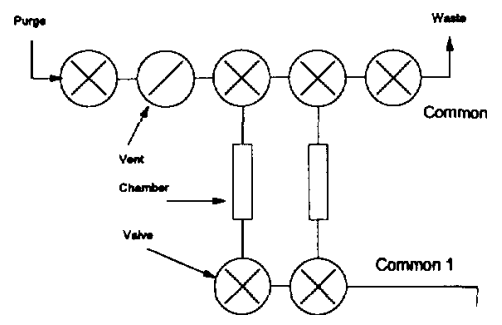

Figure 3. Sensorless fluid positioning in reaction chambers terminated with valves using vent in secondary common channel. This arrangement has improved reliability and eliminated evaporation during high-temperature operations.

result, leading and trailing fluid colors are seen to switch positions repeatedly as the fluid plug progresses through the channel. We found that after traveling five fluid-plug lengths the water appears homogeneous, apparently due to a combination of diffusion and circulation mixing. As depicted in Figure 2, this circulation effect can be explained by superimposing velocity profiles with continuity requirements. We include fluid-plug movement in our systems to exploit this effect when mixing is required. A more detailed mechanistic understanding of the observed hydrodynamics, scaling and dependence on fluid properties will be useful in future designs.

\section{RELIABLE FLUIDIC MANIPULATION}

We previously described polymeric structures for positioning, metering, and linking fluids that employ diaphragm valves and porous hydrophobic vents. We found these structures to be generally reliable for many reagent mixtures in molecular biology. Also, rounded channel cross sections have been found to produce more reliable fluid movement than square ones, particularly in the presence of surfactants.

Reagents with surfactants (such as Taq-Gold) can cause reversible blockage of the hydrophobic vents, apparently by creating a surface hydrophilic layer [13]. After such exposure the vents exhibit state-dependent behavior: they permit the free passage of gas in both directions only after gas has been forced through the membrane from the dry side. This vent-clearing step must be repeated after any subsequent contact with liquid.

Fluid positioning and linking structures with improved reliability were developed to take advantage of this behavior. For example, by terminating each chamber with a valve instead of a vent, and positioning liquids using a vent in a secondary common, evaporation has been reduced and reliability has been improved, Figure 3. This arrangement allows greater freedom in clearing the vent during fluid movement.

An improved fluid linking structure (the "T-Linker") is shown in Figure 4. The purge step (ii) provides the vent clearing necessary for reliable operation with surfactants. Hundreds of linking processes have been carried out sequentially using test solutions with as much as five times the normal amount of surfactant, with no failures.

\section{TEMPERATURE-CONTROLLED REACTION CHAMBERS}

Researchers have demonstrated that rapid thermal cycling can improve the performance of PCR reactions [14]. A system with two temperature zones using thermoelectric coolers was reported previously. Ideally, an integrated analytical system should be

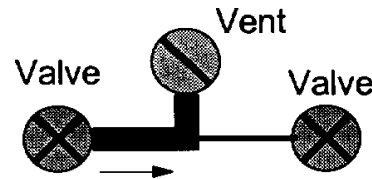

(i)

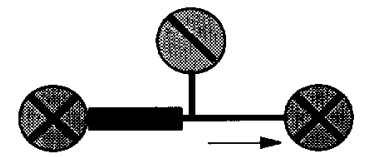

(ii)

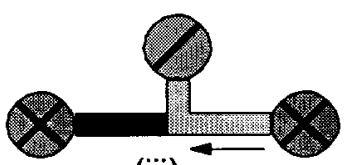

(iii)

Figure 4. Improved linking structure (T-linker) with improved reliability. Operation sequence is (i) load first reagent through left valve, (ii) purge vent through right valve, and (iii) load second reagent through right valve.

scaleable to a number of independently controlled thermal zones that can be cycled rapidly. One thermal-control method with improved ramp rate and scaleability is shown in Figure 5. Arrays of resistive heating elements with sensors are sandwiched between the reactor cartridge and a thermal insulating layer. The insulating layer is in contact with a constant cooling source (e.g. a thermoelectric cooler).

The steady state heat loss $Q$ through the thermal insulator is given by

$$
\mathrm{Q}=(\mathrm{kA} / \mathrm{x})\left(\mathrm{T}-\mathrm{T}_{\text {cooler }}\right)
$$

where $k$ and $x$ are the thermal conductivity and thickness of the thermal insulator, $A$ is the heater area, $T$ is the heater temperature, and $T_{\text {cooler }}$ is the surface temperature of the thermoelectric cooler. If we assume the thermal mass of the cartridge and reaction chamber contents are small, the time constant $\tau$ for cooling is given by

$$
\tau=\left(x^{2} C \rho\right) / k
$$

where $C$ and $\rho$ are the heat capacity and density of the thermal insulator, respectively. These equations are graphed in Figure 7 along with experimental results, assuming the thermal insulator has the properties of phenolic resin from CRC handbook: $k=0.2$ $\mathrm{W} / \mathrm{M} \mathrm{K}, \mathrm{C}=1.6 \mathrm{~J} / \mathrm{g} \mathrm{K}, \rho=1.3 \mathrm{~g} / \mathrm{cm}^{2}$, and data using the cooling time from $100^{\circ} \mathrm{C}$ to $50^{\circ} \mathrm{C}$ as $t$, while $\mathrm{T}_{\text {cooler }}=0^{\circ} \mathrm{C}$, without the used of fitting parameters [15]. Arrays of these thinfilm elements have been fabricated and PCR reactions have been successfully performed using these structures. A cooling rate exceeding $10^{\circ} \mathrm{C} / \mathrm{sec}$ after reaching steady state was demonstrated,

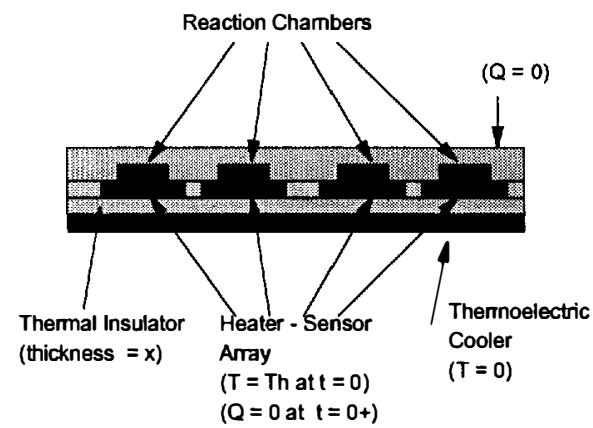

Figure 5. Thermal element array for rapid temperature cycling. Cross section schematic showing thermal elements/sensors, thermal insulator, and cooler. Boundary conditions used in simple model are in parenthesis. 


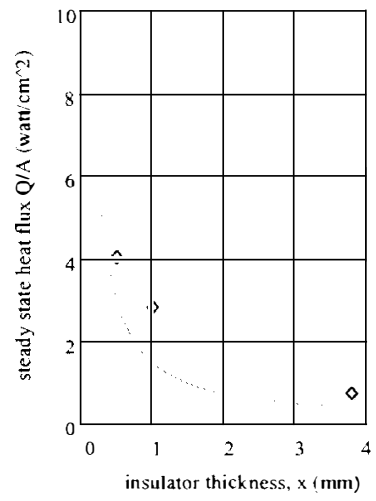

(a)

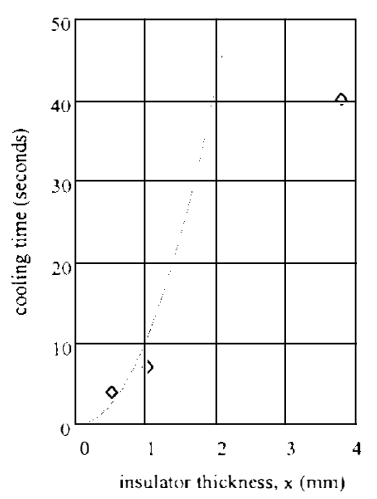

(b)
Figure 7. (a) Steady state heat flux as a function of thermal insulator thickness: theory and experiment. (b) Transient cooling versus thermal insulator thickness: theory and experiment. (Cooling through the top of the structure may explain the faster than expected rate of the $4 \mathrm{~mm}$ insulator data.)

significantly faster than other reports.

\section{CYTOCHROME P450 SYSTEM}

A multisample microfluidic system aimed at cytochrome $\mathrm{P} 450$ genotyping has been designed, fabricated, and demonstrated. The GeneChip CY P450 assay enables the rapid detection of mutations in enzymes related to drug metabolism [16]. This microfluidic system carries out a series of enzymatic reactions to prepare four separate samples for GeneChip hybridization. After isolating genomic DNA, a series of three enzymatic reactions are carried out: multiplex PCR, fragmentation and dephosphorylation (using nuclease and alkaline phosphatase), and fluorescent labeling using terminal transferase, Figure 6 [17].

The microfluidic cartridge carrics out the steps shown within the dotted box in Figure 6. Four samples are prepared simultancously in the design shown in Figure 8 for the P450 assay in a polycarbonate cartridge measuring 7 by 3.8 by $0.7 \mathrm{~cm}[18]$.

At the beginning of the proccdure, the user loads the reagent cocktails for the fragmentation and labeling reactions into storage chambers, and loads the PCR reaction mixture into the reaction

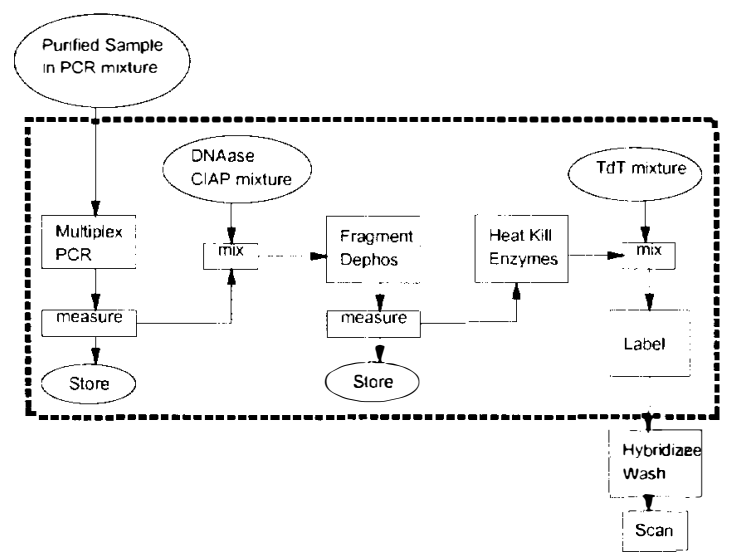

Figure 6. GeneChip CYP450 assay for mutations in the cytochrome P450 genes. The steps within the dotted box are carried out by the microfluidic system.

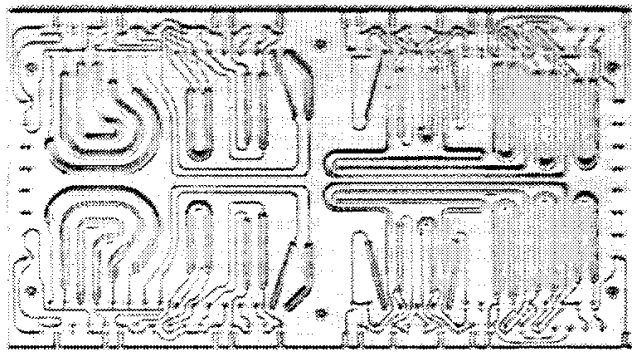

(a)

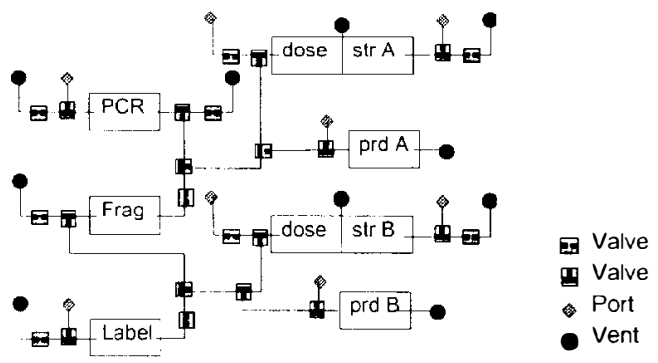

(b)

Figure 8. (a) Image of 7 by 3.8 by $0.7 \mathrm{~cm}$ polycarbonate cartridge that prepares four independent samples simultaneously (two different designs) for a GeneChip-based cytochrome P450 assay. A series of three enzymatic reactions is carried out on each sample. (b) Schematic of one sample reaction pathway.

chamber. PCR is carried out automatically, measured and mixed with the fragmentation cocktail mixture and the unused portion stored for quality control, Figure 6. After incubation in the fragmentation reaction chamber, the temperature is raised to inactivate the nuclease and alkaline phosphatase enzymes. This reaction product is measured and mixed with the labeling cocktail and the unused portion is stored for quality control. This mixture is transferred to the final reaction chamber and incubated. After the reaction is complete, the user removes the product and stored samples. Two different fluidic designs are incorporated in the cartridge; a schematic of one of these is shown in Figure 8 (a)

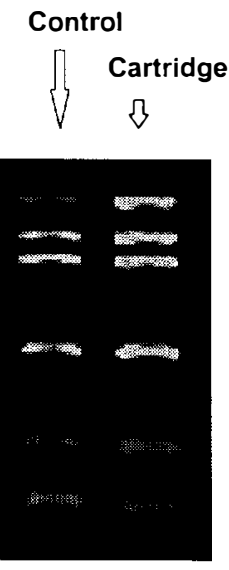

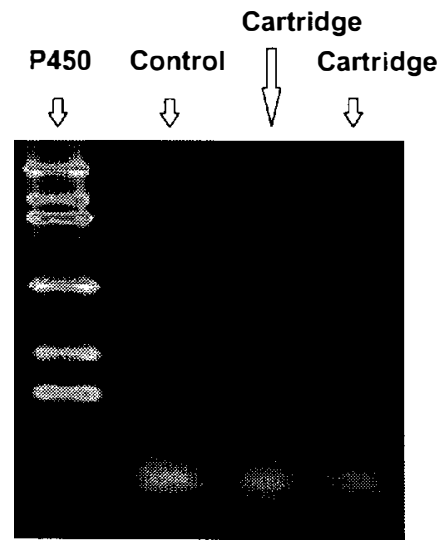

(b)
Figure 9. Agarose gel images of product from reactions carried out in test cartridges compared with control reactions carried out on a bench top. (a) Cytochrome P450 multiplex PCR. (b) DNAase fragmentation. 
Fluidic operation of this design was verified first with water, then mock reagents containing the appropriate surfactants and salts, and finally with actual samples. Thermal performance was confirmed using arrays of thermocouples mounted within the reaction chambers [19]. Each of the CYP450-assay reactions were carried out in a test cartridge, Figure 9. Sequence analysis performed using hybridization to the GeneChip array was comparable to that found using the standard bench-top samplepreparation assay.

\section{CONCLUSIONS}

We have demonstrated several enhancements to the miniaturized genetic-analysis system reported previously, including structures for improved reliability in handling fluids and scaleable thermal control. Additional enzymatic reactions have been successfully demonstrated in the cartridge-based systems, including multiplex PCR for cytochrome P450, DNAase fragmentation, and a terminal deoxy-transferase reaction. We have also introduced a unique mixing phenomenon that can be generally exploited in microfluidic systems. Automated systems to perform other multistep biochemical and chemical processes could be developed using the devices and structures described in this work.

\section{ACKNOWLEDGEMENTS}

The advice and assistance of scientists at Affymetrix were invaluable in performing these experiments. The authors would like to particularly thank Tom Ryder, Clotilde Teiling, and Weiwei Liu for validating the CYP450 results. This work was supported by NIST cooperative agreement 70NANB5H031.

\section{REFERENCES}

1 A.T. Wooley, D. Hadley, P. Landre, A.J. deMello, R.A. Mathies. M.A. Northrup, "Functional Integration of PCR Amplification and Capillary Electrophoresis in a Microfabricated DNA Analysis Device," Analytical Chemistry, 1996 68(23):4081-4086.

2 D.J. Harrison and N. Chiem, "Immunoassay Systems on Chip," Technical Digest of 1996 Solid-State Sensor and Actuator Workshop, Hilton Head Island, South Carolina, (1996), p. 5.

3 P.C. Li , D.J. Harrison, "Transport, Manipulation, and Reaction of Biological Cells On-chip using Electrokinetic Effects," Analytical Chemistry, 1997, 69(8):1564-1568.

4 P. Andersson, P. Li, Y. Ning, D. Harrison, Technical Digest of Transducers '97, International Conference on Solid-State Sensors and Actuators, Chicago (1997), p. 1311-1314.

5 R.C. Anderson, G.J. Bogdan, Z. Barniv, T.D. Dawes, J. Winkler, K. Roy, "Microfluidic Biochemical Analysis System," Technical Digest of Transducers '97, International Conference on SolidState Sensors and Actuators, Chicago (1997), p. 477-480, and

R. C. Anderson, G. J. Bogdan, and R. J. Lipshutz, "Miniaturized Genetic-Analysis System," Technical Digest of 1996 Solid-State Sensor and Actuator Workshop, Hilton Head Island, South Carolina, (1996), pp. 258-261.

6 GeneChip is a registered trademark of Affymetrix Inc.

7 M.J. Kozal, N. Shah, N. Shen, , R. Yang, R. Fucini, T. Merigan, D. Richman, D. Morris, E. Hubbell, M.S. Chee, T.G. Gingeras, Nature Medicine, 1996, 2:7:753-759.
8 D.J. Lockhart, H. Dong, M.C. Byrne, M.T. Follettie, M.V. Gallo, M.S. Chee, M. Mittmann, C. Wang, M. Kobayashi, H. Horton, E.L. Brown, Nature Biotechnology, 14:13:1675-1680, December 1996.

9 M.T. Cronin, R.V. Ficini, S.M. Kim, R.S. Masino, R.M. Wespi, C.G. Miyada, Human Mutation, 1966, 7:244-255.

10 J.G. Hacia, L.C. Brody, M.S. Chee, S.P.A. Fodor, and F.S. Collins, Nature Genetics, 14:441-447, December 14, 1996.

11 U.D. Larsen, J. Branebjerg, G. Blenkenstein, "Fast Mixing by Parallel Multilayer Lamination," Proceedings of the 2nd International Symposium on Miniaturized Total Analysis Systems $\mu T A S 96$, Basel (1996) 228, and

R. Miyake, T.S.J. Lammerink, M. Elwenspoek, J.H.J. Fluitman, “Micro Mixer with Fast Diffusion," MEMS 1993, 143.

12 Water dyed with food coloring was moved through 0.7 by 0.7 $\mathrm{mm}$ channels in polycarbonate. This effect was also observed when moving the same fluid through $1.6 \mathrm{~mm}$ id tubing.

13 Taq-gold from Perkin Elmer contains 0.5 w/\% Tween20. For example, when a Durapel (Millipore) membrane is placed in contact with a $0.5 \mathrm{w} / \%$ Tween 20 solution for 3 seconds, the originally hydrophobic surface becomes hydrophillic.

14 C.T. Wittwer, D.J. Garling, "Rapid Cycle DNA Amplification: Time and Temperature Optimization," BioTechniques, vol. 10, 1:76-83, 1991.

15 Cooling through the top of the structure may explain the faster than expected rate of the $4 \mathrm{~mm}$ insulator data.

16 C. Sachse, et al. "Cytochrome P450 2D6 variants in a Caucasian Population: Allele Frequencies and Phenotypic Consequences," Am J Hum Genet 60: 284-295, 1997.

17 Some details and modifications are described below. A full description of the reaction protocols are described in the Affymetrix product brochure "GeneChip ${ }^{\circledR}$ CYP450 Assay"

$\underline{P C R}$ Reaction 15 ul mixture includes 2 units of TaqGold polymerase and $2.5 \mathrm{mM} \mathrm{MgCl} 2$. Thermal program includes: (1) an initial denature at $95^{\circ} \mathrm{C}$ for 5 minutes, (2) a denature at $95.5^{\circ} \mathrm{C}$ for 20 seconds, (3) an anneal at $64^{\circ} \mathrm{C}$ for 20 seconds, (4) an extend at $72^{\circ} \mathrm{C}$ for 60 seconds, (5) steps 2 through 4 repeated 35 times total.

Fragmentation Reaction $15 \mathrm{ul}$ mixture includes 0.6 units DNAase, 1.5 units CIAP, and lug DNA. Thermal program consists of 20 minute incubation at $25^{\circ} \mathrm{C}$ followed by 10 minute termination at $95^{\circ} \mathrm{C}$.

Labeling Reaction 20 ul mixture includes 10 units terminal transferase, $1 \mathrm{X}$ TdTase buffer and $25 \mathrm{uM}$ FITC-ddATP.

18 Channels are 250 um deep with a 125 um radius and chambers are $0.7 \mathrm{~mm}$ deep with a $0.6 \mathrm{~mm}$ radius. Reaction chambers have 20 ul volume.

19 It was necessary to use thermocouples with 50 um diameter wires to minimize conductive heat loss along these wires. 